\title{
Automatic ICD-10 codes association to diagnosis: Bulgarian case
}

\author{
Boris Velichkov \\ bobby.velichkov@gmail.com \\ GATE Institute and Faculty of \\ Mathematics and Informatics, Sofia \\ University "St. Kliment Ohridski" \\ Sofia, Bulgaria \\ Sylvia Vassileva \\ sylvia.vassileva@gmail.com \\ Faculty of Mathematics and \\ Informatics, Sofia University "St. \\ Kliment Ohridski" \\ Sofia, Bulgaria
}

\author{
Simeon Gerginov \\ simeongerginov1@gmail.com \\ Faculty of Mathematics and \\ Informatics, Sofia University "St. \\ Kliment Ohridski" \\ Sofia, Bulgaria \\ Gerasim Velchev \\ gerasim.petrov.velchev@gmail.com \\ Faculty of Mathematics and \\ Informatics, Sofia University "St. \\ Kliment Ohridski" \\ Sofia, Bulgaria \\ Svetla Boytcheva \\ svetla.boytcheva@gmail.com \\ Institute of Information and \\ Communication Technologies, \\ Bulgarian Academy of Sciences \\ Sofia, Bulgaria
}

Panayot Panayotov

p.d.panayotov09@gmail.com

Faculty of Mathematics and

Informatics, Sofia University "St.

Kliment Ohridski"

Sofia, Bulgaria

Ivan Koychev

koychev@fmi.uni-sofia.bg

Faculty of Mathematics and

Informatics, Sofia University "St.

Kliment Ohridski"

Sofia, Bulgaria

\begin{abstract}
This paper presents an approach for the automatic association of diagnoses in Bulgarian language to ICD-10 codes. Since this task is currently performed manually by medical professionals, the ability to automate it would save time and allow doctors to focus more on patient care. The presented approach employs a fine-tuned language model (i.e. BERT) as a multi-class classification model. As there are several different types of BERT models, we conduct experiments to assess the applicability of domain and language specific model adaptation. To train our models we use a big corpora of about 350,000 textual descriptions of diagnosis in Bulgarian language annotated with ICD-10 codes. We conduct experiments comparing the accuracy of ICD-10 code prediction using different types of BERT language models. The results show that the MultilingualBERT model (Accuracy Top $1-81 \%$; Macro F1 - 86\%, MRR Top 5 - 88\%) outperforms other models. However, all models seem to suffer from the class imbalance in the training dataset. The achieved accuracy of prediction in the experiments can be evaluated as very high, given the huge amount of classes and noisiness of the data. The result also provides evidence that the collected dataset and the proposed approach can be useful in building an application to help medical practitioners with this task and encourages further research to improve the prediction

Permission to make digital or hard copies of all or part of this work for personal or classroom use is granted without fee provided that copies are not made or distributed for profit or commercial advantage and that copies bear this notice and the full citation on the first page. Copyrights for components of this work owned by others than ACM must be honored. Abstracting with credit is permitted. To copy otherwise, or republish, to post on servers or to redistribute to lists, requires prior specific permission and/or a fee. Request permissions from permissions@acm.org.

CSBio2020, November 19-21, 2020, Bangkok, Thailand

(C) 2020 Association for Computing Machinery.

ACM ISBN 978-1-4503-8823-8/20/11 . .\$15.00

https://doi.org/10.1145/3429210.3429224
\end{abstract}

accuracy of the models. By design, the proposed approach strives to be language-independent as much as possible and can be easily adapted to other languages.

\section{CCS CONCEPTS}

- Computing methodologies $\rightarrow$ Natural language processing; Neural networks; Machine learning; • Applied computing $\rightarrow$ Health informatics.

\section{KEYWORDS}

Deep Learning, BERT, ICD-10 codes association to diagnosis.

ACM Reference Format:

Boris Velichkov, Simeon Gerginov, Panayot Panayotov, Sylvia Vassileva, Gerasim Velchev, Ivan Koychev, and Svetla Boytcheva. 2020. Automatic ICD10 codes association to diagnosis: Bulgarian case. In CSBio '20: Proceedings of the Eleventh International Conference on Computational Systems-Biology and Bioinformatics (CSBio2020), November 19-21, 2020, Bangkok, Thailand. ACM, New York, NY, USA, 8 pages. https://doi.org/10.1145/3429210.3429224

\section{MOTIVATION}

The classification of diseases according to standard international classifications in medicine is an important task, because it allows mapping of terms to predefined identifiers and disambiguation of terms. This task also helps in the transformation of clinical texts into a more structured machine-readable format. On the other hand, such complex classifications with several thousand classes need to be used by medical practitioners daily. This requires comprehensive knowledge about their organization and application.

International Statistical Classification of Diseases and Related Health Problems is a classification system for medical diagnoses 
which is used for health management and clinical purposes ${ }^{1}$. This classification is used world-wide and has translations in multiple languages. The 10th edition of the classification (ICD$10)^{2}$ is used in Bulgaria by physicians when submitting outpatient records to the National Health Insurance Fund for insurance claims reimbursement. The most relevant code needs to be associated with each diagnosis. For example, "Cholera due to Vibrio cholerae 01, biovar cholerae" is associated with the ICD-10 code "A00.1". The ICD-10 classification has almost 11000 codes organized in a hierarchy and currently, most hospitals and doctors in Bulgaria have to manually assign the correct code based on the diagnosis text. The free text diagnosis often significantly differs from the description of the class. The task of the ICD-10 code association requires medical training and a lot of time which can be better spent on actual patient care. Therefore, it would be beneficial to both medical practitioners and patients if there is a way to automatically assign the correct code to a diagnosis. Our goal is to facilitate medical coding by providing the medical practitioners a shortlist of top candidate codes based on a short textual description of a diagnosis.

The ICD-10 codes association task is challenging, due to the huge amount of classes. It can be viewed as an extreme scale classification task. On the other hand, it is also a multi-class multilabel classification task, because for many diagnoses it is possible to associate more than one correct ICD-10 code.

In this research, we are focusing on the investigation of the application of deep learning approaches in the task for automatic ICD-10 codes association to diagnosis text descriptions in Bulgarian. Bulgarian is a low resource language and some natural language processing (NLP) techniques are hard to adapt. Furthermore, the amount of existing medical training data is scarce. We believe that we can benefit from some of the deep learning state of the art approaches for classification because they provide word embedding and language models as building blocks for our classification model.

This paper is structured as follows: Section 2 overviews different approaches for automatic ICD-10 codes association to clinical text, Section 3 describes the data prepared and used for the classification models training and the experiments; Section 4 presents methods investigated and developed for this study; Section 5 reports results from conducted experiments; and Section 6 concludes and sketches directions for further work.

\section{RELATED WORK}

The problem of ICD-10 code association has been explored in multiple works. One of the primary issues is the lexical variability of the diagnoses which has been addressed by incorporating knowledge bases, such as dictionaries, expanding the dataset through machine translation and working directly with meanings [1], synonyms and ontologies [19]. Automatic association of ICD-10 codes task was addressed for several languages other than English [16] like Spanish [1], Chinese [17], Dutch [7], and Turkish [11] [4]. Some preliminary work was done for Bulgarian using SVM and small training corpora [8], with achieved F-score 0.84 . We need to note that the reported results there are based on significantly smaller

\footnotetext{
${ }^{1}$ https://icd.who.int/browse10/2015/en

${ }^{2}$ https://ncpha.government.bg/bg/2019-02-19-23-22-18/icd-10
}

training and test datasets with limited number of ICD-10 classes. In this study we are trying to address a larger scope of this task, including almost all ICD-10 codes used by medical practitioners in Bulgaria.

Chen et al. [12] propose a method based on the similarity search of the longest common subsequence of text in Chinese. Unfortunately, in our case this approach is not applicable, because the majority of phrases used in the diagnosis descriptions in clinical narratives have no common words.

Another challenge is the dataset imbalance especially when trying to encode all 4-character codes as finding sufficient representatives for all classes is very difficult. Applying extreme text classification approaches to this task like splitting feature spaces or compressing label dimension helps reduce the imbalance effects [18].

A few works are tackling the classification task using information retrieval techniques, statistical classifiers and document similarity [1], as well as machine learning techniques [6]. Another approach is to view the problem as a multi-label classification task and use neural networks like CNN, LSTM/BiLSTM and HA-GRU [19], or applying BERT which has shown good results on this task in German [3]. Due to the high number of classes, combining different models into ensemble has shown slight improvement in the results [3]. Hierarchical approaches were proposed in [17] and [10]. Dictionary lookup based approach was explored in [14]. In [6] a system based on machine learning (ML) approaches is presented. The authors report results for a subset of 1500 ICD-10 classes with achieved $92 \%$ precision for the primary disease. Arifoglu et al.[4] propose a recommendation system CodeMagic that proposes top $\mathrm{K}$ possible ICD-10-AM codes associated with patient records. This is one of the possible solutions for the multi-label classification task especially when there is lack of context around the current diagnosis. In our approach we are using top 5 proposed classes as an evaluation result of the proposed deep learning approach.

\section{DATA}

\subsection{Diagnoses and ICD-10 codes}

In their article, Boytcheva et al. [9] present methods for automatic generation of corpora that contain descriptions of diagnoses in Bulgarian and their associated codes in ICD-10-CM (International Classification of Diseases, 10th revision, Clinical Modification). In addition, they have generated a public dataset which we enhance and use in this paper ${ }^{3}$. The proposed approach in [9] is based on two types of data: open data and Linked Open Data.

The main open data resource is the ICD-10-CM translated in Bulgarian ${ }^{4}$. It contains about 11,000 classes organized in 22 toplevel categories, 211 subcategories at level 2, 2065 at level 3 and 8946 at level 4. Each category in the hierarchy is shown in Table 1. For the purposes of our article we consider the level 3 and level 4 subcategories.

\footnotetext{
${ }^{3}$ https://github.com/BorisVelichkov/ICD10-Medical-Data

${ }^{4}$ http://www.zdrave.bg/normativi/MKB10.pdf
} 
Table 1: Example of ICD-10 Categories with English description.

\begin{tabular}{lll}
\hline Level & $\begin{array}{l}\text { ICD-10 } \\
\text { Code }\end{array}$ & Diagnosis Description (English) \\
\hline 1 & A & Certain infectious and parasitic diseases \\
\hline 2 & A0 & Intestinal infectious diseases \\
\hline 3 & A00 & Cholera \\
\hline 4 & A00.0 & $\begin{array}{l}\text { Cholera due to Vibrio cholerae 01, biovar } \\
\text { cholerae (Classical cholera) }\end{array}$ \\
\hline
\end{tabular}

Two additional resources include the golden standard (GS) developed in [8] and the official document of the International Classification of Diseases: "ICD-10-CM Alphabetical Index"5,6.

The Linked Open Data resource consists primarily of data collected from Wikidata ${ }^{7}$. In addition Machine translation is applied from English to Bulgarian, from English to Latin, and transliteration of the resulting dataset from Latin to Cyrillic ${ }^{8}$.

The resulting corpus consists of about 189,254 pairs of 3-character ICD-10 codes with 2035 unique codes and 377,939 pairs of 4character ICD-10 codes with 11064 unique codes.

\subsection{Balancing and Augmenting the Data}

As described in [9], the classes in the dataset are imbalanced, which in turn can affect the accuracy of the classification algorithms.

In order to address the class imbalance problem, we analyzed the dataset and found that there are 4939 classes which have less than 5 samples in the dataset. Since these classes do not have sufficient representation, we enhance the dataset using several different approaches.

For the classes which are under-represented (less than 5 samples) and which have a 4-character code, we assign the existing samples to their parent class and remove the 4-character specific class from the classification. For ex., "менингеал туберкулом" ("meningeal tuberculoma") which belongs to class A17.1 is assigned to class A17. This strengthens the parent classes and improves the imbalance. In addition, 405 classes which are under-represented are encoded using 3 characters, so we cannot apply the first approach. We further enhance these classes using data augmentation techniques:

- swap random letters within a single word

- delete random letter within a single word

- replace a random letter with a letter close to it on the keyboard

For each sample selected for augmentation a single technique is applied once only if the diagnosis consists of more than 2 words. These techniques simulate small typos which can happen when the practitioner is writing a diagnosis in a hurry. The samples are augmented using the nlpaug library ${ }^{9}$. A total of 361 records are added to the dataset which represents $0.1 \%$ of the dataset size and thus would not increase the noise in the dataset significantly.

\footnotetext{
${ }^{5}$ https://icd.codes/icd10cm/alphabetical-index

${ }^{6} \mathrm{https} / / /$ github.com/BorisVelichkov/icd10-msword-extraction

${ }^{7}$ https://www.wikidata.org

${ }^{8}$ https://github.com/BorisVelichkov/latin-transliterator

${ }^{9}$ https://pypi.org/project/nlpaug/
}

In addition, class trimming is applied to reduce the number of under-represented classes without losing data. Based on analysis of the different ICD-10 codes, we found that codes starting with the letters V-Y and Z do not contain disease diagnosis but have a special purpose to capture external factors influencing health. Table 2 shows the descriptions of these categories. Since classification of these categories would not have a lot of value for a General Practitioner, we have assigned all of the samples from categories V01-Y98 to the top level V category and all Z00-Z99 to the top level $\mathrm{Z}$ category, following the ICD-10 grouping logic.

Table 2: V-Y and Z Category Descriptions.

\begin{tabular}{ll}
\hline $\begin{array}{l}\text { ICD-10 } \\
\text { Category }\end{array}$ & Description (English) \\
\hline V01-Y98 & External causes of morbidity and mortality \\
\hline Z00-Z99 & $\begin{array}{l}\text { Factors influencing health status and contact } \\
\text { with health services }\end{array}$ \\
\hline
\end{tabular}

Apart from under-represented classes, we have one group of classes which is over-represented with almost 80,000 samples the group of $\mathrm{Q}$ codes. The biggest offender is the $\mathrm{Q} 87$ code which contains a big list of rare diseases with unique names. In the dataset provided by [9], some diagnosis samples have more than one valid class including a specific 4 -character class and the more general 3 -character parent. In order to avoid duplication of the diagnoses, we remove any duplicate diagnosis texts from the parent level code for Q87 as they appear in their respective 4-character class as well. Thus we reduce the $Q$ code samples to almost 68,000 without losing the data for these diagnoses.

The final distribution of the classes after augmentation can be seen in Fig. 1. After applying the changes to the dataset, the number of classes for the classification task is reduced to 5854 and the total number of samples in the dataset is 345,591 . Fig. 2 shows the number of classes grouped by the sample size in the original and augmented datasets. We have significantly reduced the number of classes which have less than 10 samples from 6310 to 2538 .

\subsection{Language Model Fine-Tuning Dataset}

In addition to the text classification dataset, we also gather a fine-tuning dataset from crawled medical articles from different medical websites in Bulgarian: Framar ${ }^{10}$, BGMedic ${ }^{11}$ and Puls ${ }^{12}$. The crawled medical articles are combined to form the dataset that is used for the fine-tuning of BERT. Some cleaning and pre-processing of the crawled data is performed, including special characters and newlines removal.

All of the crawled medical articles describe diseases with their symptoms, causes and possible treatment. From "Framar" we have crawled 9519 articles, each containing 491 words on average. From "BGMedic" we have crawled 236 articles containing 528 words on average, and from "Pulse" - 473 articles and 1360 words on average.

\footnotetext{
${ }^{10}$ https://www.framar.bg/

${ }^{11} \mathrm{https} / / /$ www.bgmedic.com/

${ }^{12}$ https://www.puls.bg/
} 


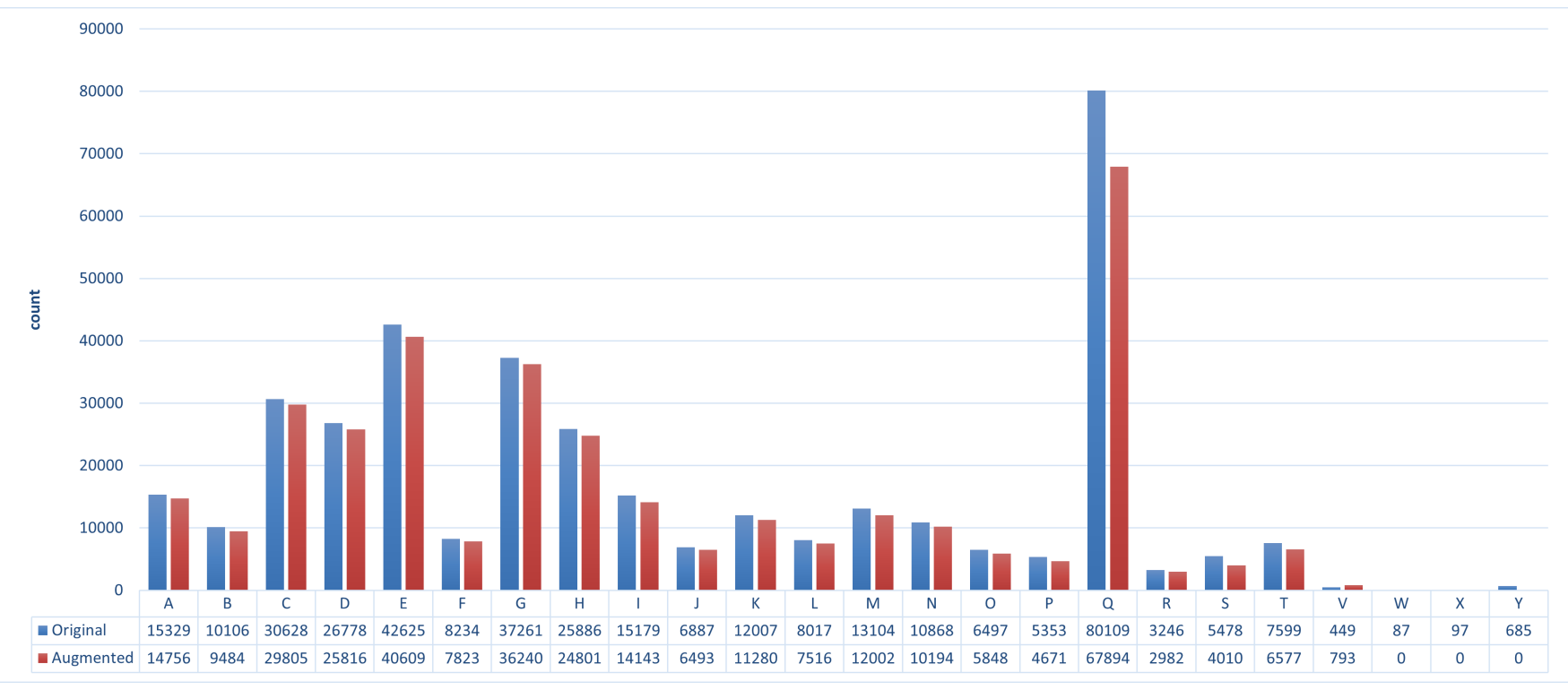

Figure 1: Distribution of the diagnoses per ICD-10 classes in the combined dataset.

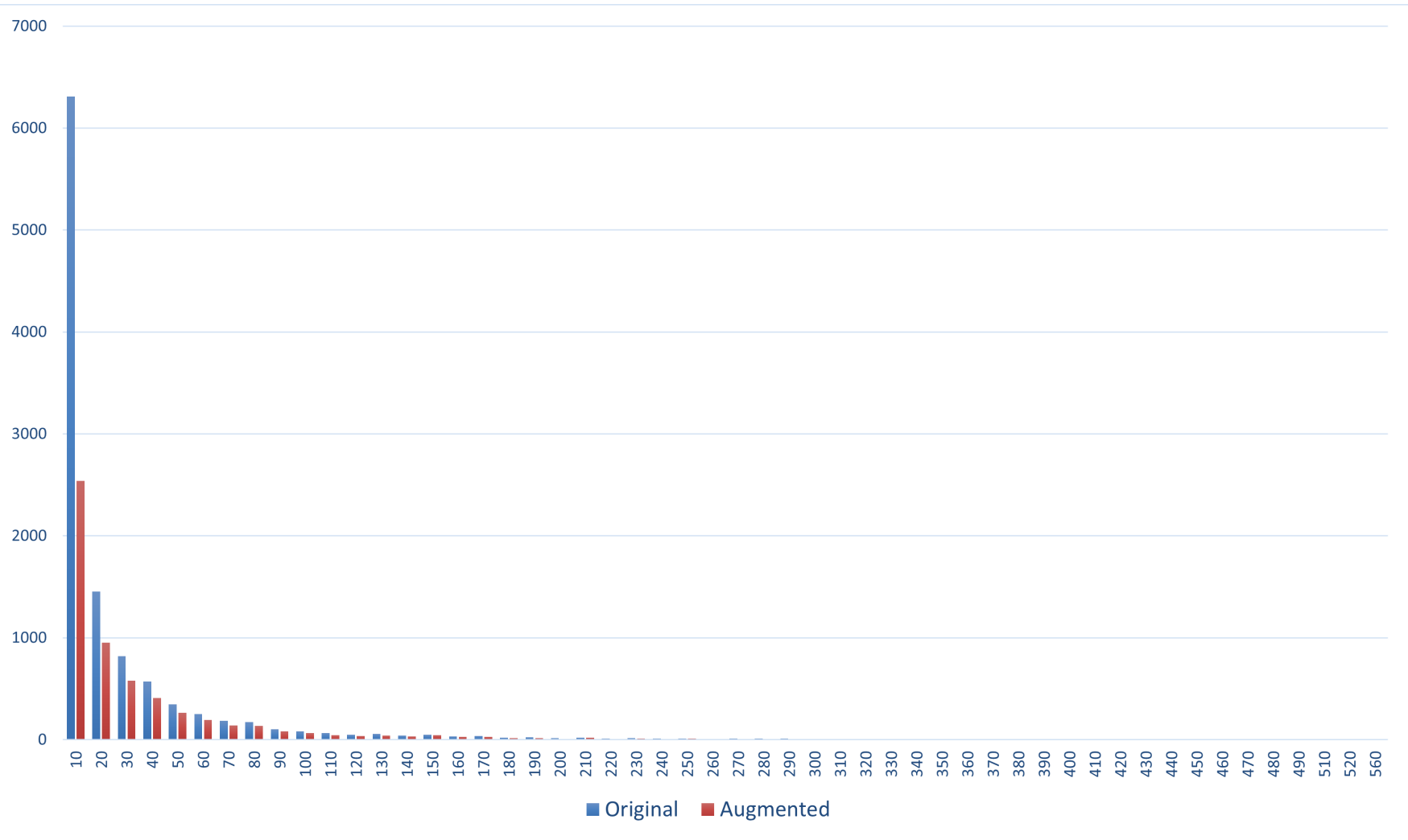

Figure 2: Number of classes grouped by sample size in the original and augmented datasets. 


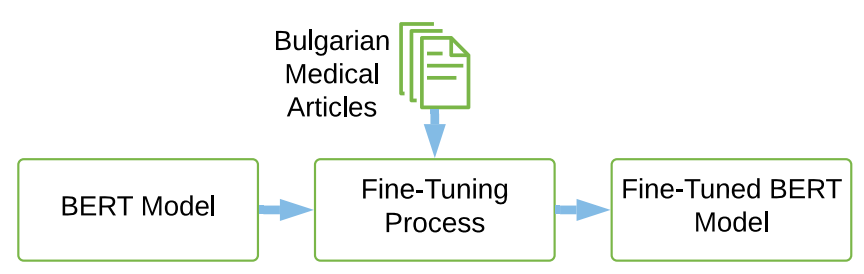

Figure 3: Pipeline for fine-tuning BERT models using the Bulgarian Medical Articles dataset.

The resulting datasets are published and open for public usage $^{13,14}$.

\section{METHODS}

We are applying BERT for text classification to solve a multi-class classification problem ${ }^{15}$. We provide the top 5 predictions in order to allow the system to return more than one relevant code for each diagnosis.

We apply BERT fine-tuning for text classification in order to match the correct ICD-10 code to the input diagnosis.

BERT (Bidirectional Encoder Representations from Transformers) [13] is a context-aware word representation model based on masked language model and fine-tuned using bidirectional transformers. BERT has been successfully used in a wide variety of NLP tasks including text classification. With additional fine-tuning specific to the NLP task, BERT has been able to achieve state-of-the-art results.

Due to BERT's successful applications across many tasks, there exist multiple language models which are language or domainspecific. We explore several of these models and their application towards the diagnosis classification in Bulgarian.

We run a fine-tuning task for all of these models using a corpus of medical articles in Bulgarian in order to improve the contextual awareness for Bulgarian medical terms. After that we fine-tune the original models as well as the fine-tuned ones for the text classification task in order to compare the results.

\subsection{Fine-Tuning of the BERT Models}

We fine-tune the base BERT models over a corpus of Bulgarian medical articles with disease descriptions. The corpus consists of 10,000 articles and we split it to use $80 \%$ for training, $10 \%$ for hyper-parameter fine-tuning and $10 \%$ for testing.

For the fine-tuning we run the masked language task as described in the original BERT paper [13]. We use the Simple Transformers library, which is based on HuggingFace Transformers ${ }^{16}$. We use 15\% masking of the words in the text and BERT is trained to recognize the masked words. The perplexity metric on the test set is reported

In NLP the Perplexity (PP) is a measurement of how well the language model predicts a test sample. Formally it can be defined as follows:

$$
P P(W)=P\left(w_{1} w_{2} \ldots w_{N}\right)^{-\frac{1}{N}}
$$

\footnotetext{
${ }^{13}$ https://github.com/BorisVelichkov/scrapping-framar-and-bgmedic

${ }^{14} \mathrm{https} / /$ github.com/BorisVelichkov/scrapping-puls

${ }^{15} \mathrm{https} / /$ github.com/BorisVelichkov/icd10-slavic-multilingual-bert

${ }^{16}$ https://github.com/ThilinaRajapakse/simpletransformers
}

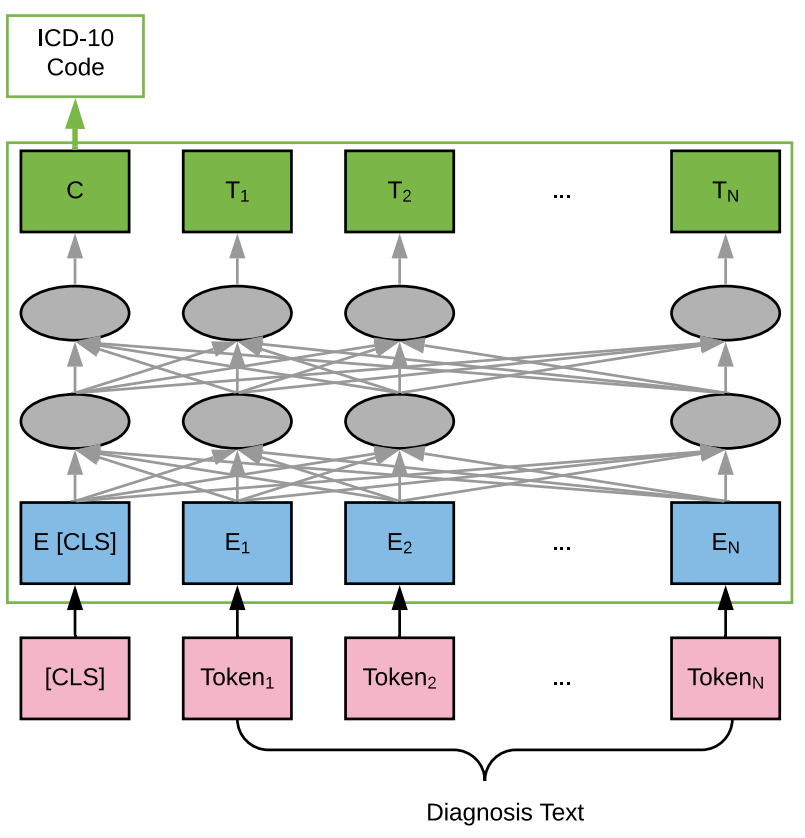

Figure 4: Fine-tuning BERT for text classification architecture.

where $W=w_{1} w_{2} \ldots w_{N}$ is a test set and $N$ is the number of words ${ }^{17}$.

For tokenization, we use WordPiece tokenization and the original vocabulary as defined in each model. Any new words can be represented by existing tokens in the vocabulary. Using a domainspecific vocabulary requires a bigger corpus for training which is unavailable for Bulgarian language. Training is performed using the following configuration: learning rate $-4 \mathrm{e}-5$, batch size -8 , max epochs -12 . The epoch with lowest score on perplexity was selected and used for further experiments.

\subsection{Fine-Tuning BERT for ICD-10 Classification Task}

In order to fine-tune BERT for our text classification task, we use the architecture established by Devlin et al. [13].

Each class in ICD-10 is assigned a number and the model is trained to classify over the numerical value of each class. For the Bulgarian ICD-10 classification, we have reduced the number of classes to 5854. The text classification corpus consists of 345,591 diagnoses and their corresponding ICD-10 codes. We apply data pre-processing on the diagnosis text by stemming using bulstempy $^{18}$ and removing the stop words using the BTB list ${ }^{19}$. We split the dataset using stratification and assign $80 \%$ for training, $10 \%$ for hyper-parameter tuning and $10 \%$ for testing using scikit-learn library $^{20}$.

As input for the fine-tuning procedure we tokenize the input diagnosis and prepend and append the special tokens [CLS] and

\footnotetext{
${ }^{17} \mathrm{https} / /$ towardsdatascience.com/perplexity-intuition-and-derivation105dd481c8f3

${ }^{18} \mathrm{https} / / /$ github.com/mhardalov/bulstem-py

${ }^{19} \mathrm{http}$ ///bultreebank.org/wp-content/uploads/2017/04/BTB-StopWordList.zip

${ }^{20}$ https://scikit-learn.org/stable/
} 
Table 3: BERT models used in experiments with their fine-tuning corpora.

\begin{tabular}{ll}
\hline BERT model & Fine-Tuned on Corpus \\
\hline MultilingualBERT & BERT-base with Wikipedia articles in 104 languages incl. Bulgarian [13] \\
SlavicBERT & Multilingual with Wikipedia articles and news in Bulgarian, Czech, Polish and Russian [5] \\
BioBERT-Base v1.1 & BERT-base-cased and PubMed Abstracts 1 M [15] \\
ClinicalBERT & BioBERT-Base v1.0 (+PubMed 200K+ PMC270K) [2] with 2 million clinical notes in English \\
\hline
\end{tabular}

[SEP] around it, as described in the original BERT paper [13] [CLS] is a fixed-dimensional word embedding which represents the concept of the input diagnosis and is used for the classification. A linear layer was added for the final classification and the probability for each class was computed using softmax function. The model was trained on the classification dataset for a maximum of 10 epochs. The model produces the top 5 ICD-10 codes with highest probability as predictions for the input diagnosis class.

ICD-10 is a hierarchical classification system and all 4-character codes are sub-classes of a 3-character parent class, for ex. A00.1 is a subclass of A00. In our training dataset all instances of 4-character diagnosis also appear as their 3-character parent class. Therefore, for each diagnosis text, we can also accept multiple codes as correct with the most specific 4-character code being the most accurate. For example, for the diagnosis "endometrial stromal sarcoma" the valid codes are C54 and C54.1. Using the latter definition of accepting multiple correct codes, we report the accuracy of top 1 predictions as well as the mean reciprocal rank in the top 5 predicted classes. In order to account for the class imbalance we also report the macro-average F1 score.

Accuracy (A) is the percentage of test samples which were correctly classified by the model, where y_true are the correct classes, y_pred are the predicted classes and $\mathrm{N}$ is the number of samples:

$$
A\left(y \_ \text {pred }, y \_ \text {true }\right)=\frac{\sum_{i=1}^{N}\left[y_{-} \text {pred }_{i}=y_{-} \text {true }_{i}\right]}{N}
$$

where

$$
\left[y_{i}=y_{j}\right]= \begin{cases}0 & \text { if } y_{i} \neq y_{j} \\ 1 & \text { if } y_{i}=y_{j}\end{cases}
$$

The formula to calculate mean reciprocal rank (MRR), where $Q$ is the number of test samples and rank is the rank position of the first relevant document ${ }^{21}$ :

$$
M R R=\frac{1}{Q} \sum_{i=1}^{Q} \frac{1}{\text { rank }_{i}}
$$

The macro-average F1 score calculates the F1 score for each class and finds the unweighted average. The F1 score was calculated using the following formula, where $\mathrm{C}$ is the number of classes and $\mathrm{F} 1_{\mathrm{i}}$ is the $\mathrm{F} 1$ score for class $\mathrm{i}^{22}$ :

$$
F 1_{\text {macro }}=\frac{1}{C} \sum_{i=1}^{C} F 1_{i}
$$

The F1 score for a single class is the harmonic mean of precision and recall for that class. Precision and recall are defined using

\footnotetext{
${ }^{21}$ https://en.wikipedia.org/wiki/Mean_reciprocal_rank

${ }^{22}$ https://en.wikipedia.org/wiki/F1_score
}

Table 4: BERT models and fine-tuning results.

\begin{tabular}{ll}
\hline BERT model & Fine-tuned model perplexity \\
\hline MultilingualBERT & 1.7747 \\
SlavicBERT & 2.9527 \\
BioBERT & 1.2146 \\
ClinicalBERT & 1.4613 \\
\hline
\end{tabular}

the number of correctly predicted samples for the class (tp - true positive), the number of samples incorrectly predicted to belong to the class (fp - false positive) and the number of samples actually belonging to the class but predicted to belong to another class ( $\mathrm{fn}$ false negative) ${ }^{23}$ :

$$
\begin{gathered}
\text { Precision }=\frac{t p}{t p+f p} \\
\text { Recall }=\frac{t p}{t p+f n} \\
F 1=2 * \frac{\text { precision } * \text { recall }}{\text { precision }+ \text { recall }}
\end{gathered}
$$

We run preliminary experiments on the original dataset from [9] as well as using the augmented dataset we have created. We explore different hyper-parameters for learning rate, batch size and number of training epochs and selected the model with highest accuracy and mean reciprocal rank.

\section{EXPERIMENTS AND RESULTS}

As a baseline we perform several experiments using classical classification approaches and achieved the following results after applying ANOVA for features selection:

- Logistic regression (Lasso (L1), C=1) - with best achieved results $\mathrm{F} 153.36 \%$, Precision $71.67 \%$ and Recall $49.23 \%$;

- Random Forest (Number of trees: 15, Maximal number of considered features: 3, Replicable training, unlimited Maximal tree splitting nodes with maximum instances: 3 ) - with best achieved results F1 62.03\%, Precision $63.96 \%$ and Recall $61.79 \%$;

- SGD - with best achieved results F1 50.42\%, Precision 67.28\%, and Recall 50.78\%.

The experiments described in this section aim to evaluate and compare different BERT models. 
Table 5: BERT models and classification results.

\begin{tabular}{llll}
\hline BERT model & Accuracy & Macro F1 & MRR \\
\hline MultilingualBERT & 0.77 & 0.84 & 0.86 \\
BioBERT & 0.69 & 0.72 & 0.81 \\
ClinicalBERT & 0.63 & 0.42 & 0.70 \\
\hline $\begin{array}{l}\text { MultilingualBERT- } \\
\text { Fine-tuned }\end{array}$ & 0.81 & 0.86 & 0.88 \\
$\begin{array}{l}\text { BioBERT-Fine- } \\
\text { tuned }\end{array}$ & 0.74 & 0.77 & 0.84 \\
\hline
\end{tabular}

\subsection{Fine-Tuning of the BERT Models}

The results from fine-tuning the different BERT models are displayed in Table 4. BioBERT appears to have lowest perplexity over the test set even though it was trained only on English biomedical articles. SlavicBERT is showing highest perplexity scores and was thus eliminated from further experiments. Biomedical terminology often has Latin or Greek origin and this could explain why the SlavicBERT model trained specifically on Slavic languages does not show very good results on this task. MultilingualBERT model has slightly higher perplexity than the domain-specific models as it has been trained on a broad range of languages and domains from Wikipedia.

\subsection{Fine-Tuning BERT for ICD-10 Classification Task}

Preliminary experiments performed using the original dataset from [9] show highest accuracy of $51 \%$ and MRR of $67 \%$ for the MultilingualBERT models. BioBERT and ClinicalBERT are 2-3\% behind which is not statistically significant. Evaluation on the augmented dataset shows significant improvement over the original dataset and the results are displayed in Table 5 . The best results are achieved by the fine-tuned MultilingualBERT trained with the following configuration: learning rate $-1 \mathrm{e}-4$, batch size -64 , best epoch - 10. All other models showed best results using learning rate - 5e-5, batch size - 16. Best epoch recorded was 10 for all models.

Results from experiments show an advantage for the MultilingualBERT models (Accuracy - 81\%, Macro F1 - 86\%, MRR - 88\%) and BioBERT is not far behind. ClinicalBERT performs with significantly less accuracy with highest of $63 \%$. When reviewing the MRR, BioBERT comes even closer to the MultilingualBERT models, signifying that it is able to predict correct classes in its second to fifth prediction.

Table 5 shows a few sample diagnoses and their predicted and true classes. The samples are selected to represent one underrepresented class in the dataset (E58), one over-represented class which contains a lot of rare disease names (Q87.8) and one diagnosis which corresponds to 4 different classes. We provide top 5 predictions to the practitioner and can display a short description of each class so that they can select the class which best fits their case.

The reported preliminary results do not provide evidence that the fine-tuning of the models can increase or decrease the accuracy. Further experiments are needed to explore the effect and

\footnotetext{
${ }^{23}$ https://en.wikipedia.org/wiki/Precision_and_recall
}

Table 6: Model predictions for 3 sample diagnoses.

\begin{tabular}{|c|c|c|}
\hline $\begin{array}{l}\text { Diagnosis Text (Bulgarian and } \\
\text { English) }\end{array}$ & $\begin{array}{l}\text { Predicted } \\
\text { Classes }\end{array}$ & $\begin{array}{l}\text { True } \\
\text { Classes }\end{array}$ \\
\hline $\begin{array}{l}\text { "нарушени нару абсорбци } \\
\text { калци" ("dietary calcium defi- } \\
\text { ciency") }\end{array}$ & $\begin{array}{l}\text { E58, } \\
\text { E83.5, } \\
\text { E83, } \\
\text { M11, } \\
\text { E87 }\end{array}$ & E58 \\
\hline $\begin{array}{l}\text { "синдром лабанд" ("Laband } \\
\text { syndrome") }\end{array}$ & $\begin{array}{l}\text { Q87.0, } \\
\text { Q04, } \\
\text { Q04.3, } \\
\text { Q87.8, } \\
\text { Q87.3 }\end{array}$ & $\begin{array}{l}\text { Q87.8, } \\
\text { Q87 }\end{array}$ \\
\hline $\begin{array}{lr}\text { "малигнум } & \text { гландуларум } \\
\text { ендокринарум } & \text { тумор } \\
\text { панкреат" ("Malignant neo- } \\
\text { plasm of endocrine pancreas") }\end{array}$ & $\begin{array}{l}\text { C25, } \\
\text { C25.4, } \\
\text { D13.7, } \\
\text { D13, } \\
\text { E16 }\end{array}$ & $\begin{array}{l}\text { D13, } \\
\text { D13.7, } \\
\text { C25, } \\
\text { C25.4 }\end{array}$ \\
\hline
\end{tabular}

usability of the fine-tuning phase. Overall, BERT is successfully classifying the diagnosis and is able to predict a relevant code. Both MultilingualBERT models are scoring on MRR slightly better than BioBERT and significantly better than ClinicalBERT showing the slight advantage of MultilingualBERT's language-specific training.

With further modifications to the training task, we could explore the accuracy of finding the most specific code matching the diagnosis.

Due to the imbalanced nature of the training dataset, some classes have very small representation while others have disproportionately more samples and thus appear with higher probability in the model predictions.

The confusion matrix of wrongly predicted classes for each major group shows the highest confusion between classes within the same group, which signifies the model has difficulty distinguishing between minor differences in the diagnosis. In addition, the $\mathrm{Q}$ class has the highest number of confusions as it is the most represented class in the dataset.

The custom fine-tuned models show insignificantly higher scores from the original base models which could be due to the small language model fine-tuning dataset and the fact that we are using the original model vocabulary instead of extending it. Further fine-tuning using custom vocabulary could be explored to improve the fine-tuned language models' performance.

The results for MultilingualBERT and Fine-tuned MultilingualBERT outperform all classical classification models, which are used as baseline. Although the accuracy of these BERT models is comparable with the previous work for Bulgarian[8] we need to note that there is significant difference between the size and the scope of these two studies. The size of the training and test datasets in this research is about 5 times larger than the previous one, and the number of the ICD-10 classes used for the classification task here is larger as well. The same is valid also for other related work - the reported results for other languages are for smaller training datasets and limited number of ICD-10 classes. 


\section{CONCLUSION AND FURTHER WORK}

In this article we present an approach for Bulgarian language diagnosis to ICD-10 code association. We are applying BERT for text classification to provide the top 5 predictions in order to allow the system to return more than one relevant code for each diagnosis. BERT classifier successfully handles the large amount of classes. However, due to dataset imbalance and parent-class bias, the model has higher success in recognizing parent classes rather than the most specific relevant class.

The fine-tuned language model does not show any improvement in the classification task signifying it has a hard time learning Bulgarian medical terms without extending the vocabulary. Further work can explore additional fine-tuning of BERT models with extending their vocabulary to include medical-specific Bulgarian vocabulary and phrases.

\section{ACKNOWLEDGMENTS}

This research is partially funded by the Bulgarian Ministry of Education and Science, grant DO1-200/2018 'Electronic health care in Bulgaria' (e-Zdrave) and the Bulgarian National Science Fund, grant DN-02/4-2016 'Specialized Data Mining Methods Based on Semantic Attributes' (IZIDA). We are grateful to anonymous reviewers for useful comments and suggestions.

\section{REFERENCES}

[1] Mario Almagro, Raquel Martinez, Victor Fresno, and Soto Montalvo. 2020. ICD-10 Coding of Spanish Electronic Discharge Summaries: An Extreme Classification Problem. IEEE Access (2020).

[2] Emily Alsentzer, John R Murphy, Willie Boag, Wei-Hung Weng, Di Jin, Tristan Naumann, and Matthew McDermott. 2019. Publicly available clinical BERT embeddings. arXiv preprint arXiv:1904.03323 (2019).

[3] Saadullah Amin, Günter Neumann, Katherine Dunfield, Anna Vechkaeva, Kathryn Chapman, and Morgan Wixted. 2019. MLT-DFKI at CLEF eHealth 2019: Multilabel Classification of ICD-10 Codes with BERT.

[4] Damla Arifoğlu, Onur Deniz, Kemal Aleçakır, and Meltem Yöndem. 2014 CodeMagic: semi-automatic assignment of ICD-10-AM codes to patient records. In Information Sciences and Systems 2014. Springer, 259-268.

[5] Mikhail Arkhipov, Maria Trofimova, Yurii Kuratov, and Alexey Sorokin. 2019. Tuning multilingual transformers for language-specific named entity recognition.
In Proceedings of the 7th Workshop on Balto-Slavic Natural Language Processing. 89-93.

[6] Aitziber Atutxa, Alicia Pérez, and Arantza Casillas. 2017. Machine learning approaches on diagnostic term encoding with the ICD for clinical documentation. IEEE journal of biomedical and health informatics 22, 4 (2017), 1323-1329.

[7] Ayoub Bagheri, Arjan Sammani, Peter GM Van der Heijden, Folkert W Asselbergs, and Daniel L Oberski. 2020. Automatic ICD-10 classification of diseases from Dutch discharge letters. In BIOINFORMATICS 2020-11th International Conference on Bioinformatics Models, Methods and Algorithms, Proceedings; Part of 13th International foint Conference on Biomedical Engineering Systems and Technologies, BIOSTEC 2020, Vol. 13. SciTePress, 281-289.

[8] Svetla Boytcheva. 2011. Automatic matching of ICD-10 codes to diagnoses in discharge letters. (2011), 11-18.

[9] Svetla Boytcheva, Boris Velichkov, Gerasim Velchev, and Ivan Koychev. 2020. Automatic Generation of Annotated Corpora of Diagnoses with ICD-10 codes based on Open Data and Linked Open Data. In 2020 Federated Conference on Computer Science and Information Systems (FedCSIS). IEEE.

[10] Finneas Catling, Georgios P Spithourakis, and Sebastian Riedel. 2018. Towards automated clinical coding. International journal of medical informatics 120 (2018), $50-61$.

[11] Nefise Meltem CEYLAN, Adil ALPKOÇAK, and Afsun Ezel ESATOĞLU. 2012. Tıbbi Kayıtlara ICD-10 Hastalık Kodlarının Atanmasına Yardımcı Akıllı Bir Sistem. (2012)

[12] YunZhi Chen, HuiJuan Lu, and LanJuan Li. 2017. Automatic ICD-10 coding algorithm using an improved longest common subsequence based on semantic similarity. PloS one 12, 3 (2017), e0173410.

[13] Jacob Devlin, Ming-Wei Chang, Kenton Lee, and Kristina Toutanova. 2018. Bert: Pre-training of deep bidirectional transformers for language understanding. arXiv preprint arXiv:1810.04805 (2018).

[14] Jitendra Jonnagaddala and Feiyan Hu. 2017. Automatic Coding of Death Certificates to ICD-10 Terminology.. In CLEF (Working Notes).

[15] Jinhyuk Lee, Wonjin Yoon, Sungdong Kim, Donghyeon Kim, Sunkyu Kim, Chan Ho So, and Jaewoo Kang. 2020. BioBERT: a pre-trained biomedical language representation model for biomedical text mining. Bioinformatics 36, 4 (2020), 1234-1240.

[16] Aurélie Névéol, Hercules Dalianis, Sumithra Velupillai, Guergana Savova, and Pierre Zweigenbaum. 2018. Clinical natural language processing in languages other than english: opportunities and challenges. Fournal of biomedical semantics 9, 1 (2018), 12 .

[17] Wenxin Ning, Ming Yu, and Runtong Zhang. 2016. A hierarchical method to automatically encode Chinese diagnoses through semantic similarity estimation. $B M C$ medical informatics and decision making 16, 1 (2016), 1-12.

[18] Bekir Parlak and Alper Kursat Uysal. 2018. On feature weighting and selection for medical document classification. In Developments and Advances in Intelligent Systems and Applications. Springer, 269-282.

[19] Qiong Wang, Zongcheng Ji, Jingqi Wang, Stephen Wu, Weiyan Lin, Wenzhen $\mathrm{Li}$, Li Ke, Guohong Xiao, Qing Jiang, Hua Xu, and Yi Zhou. 2020. A study of entity-linking methods for normalizing Chinese diagnosis and procedure terms to ICD codes. Fournal of Biomedical Informatics 105 (04 2020), 103418. https://doi.org/10.1016/j.jbi.2020.103418 